\title{
Revisiting the Concept of Nuclear Rotation: Quantum and Classical Viewpoints
}

\author{
Makito Oi \\ Institute of Natural Sciences, Senshu University, \\ 3-8-1 Kanda-Jinbocho, Chiyoda-ku, Tokyo 101-0051, Japan
}

Received 31 December 2021

doi: https://doi.org/10.55318/bgjp.2022.49.1.097

\begin{abstract}
Nuclear rotation is revisited to seek for a new perspective. Review is made first for the basic concept of the nuclear rotation. Then, the quantization procedure of the rigid-body Hamiltonian is reviewed for a quantum mechanical understanding of the dynamics of rigid-body rotations. A linear rigid body is then investigated and its motion is studied from classical and quantum viewpoints. Finally, an application of the linear rigid-body rotation is attempted for a description of elongated nuclear systems, such as Beryllium 8, a two-alpha-cluster system.
\end{abstract}

KEY WORDS: ENTER PLEASE KEY WORDS OR PHRASES IN ALPHABETICAL ORDER, SEPARATED BY COMMAS.

\section{Introduction}

In the beginning of the Bohr-Mottelson textbook [1], a Hamiltonian is presented

$$
H=H_{\text {intr }}(\boldsymbol{p}, \boldsymbol{q})+H_{\mathrm{coll}}(\boldsymbol{I}, \boldsymbol{\Omega}),
$$

so as to imply that nuclear many-body problem can be decomposed into the intrinsic and collective degrees of freedom. If so, we can split our investigations to a dynamics of constituent particles in the mean field and the rigid-rotor kinematics, which can greatly simplify our theoretical efforts in understanding nuclear structure. The rigid-body part, corresponding to the collective degrees of freedom, can be handled through a quantization of the classical rigid-body Hamiltonian,

$$
H_{\text {coll }}=\sum_{k=1}^{3} \frac{I_{k}^{2}}{2 \mathcal{J}_{k}},
$$

in which $I_{k}$ and $\mathcal{J}_{k}$ are angular momentum and moment of inertia, respectively. Nuclear system has attracted physicists for its diversity: it can be superfluid or superconductive materials, crystals of alpha clusters, and a rigid-body object 
with a well-bounded surface. In the early stage of the nuclear-physics investigations, scientists tried to understand what nuclear systems are really like. Varieties of models, such as the Fermi gas model, the liquid drop model, the shell model, etc, were presented and reconciled with each other to unveil more elaborate and profound facet of the nuclear systems. The nuclear rotational model was also proposed in the quite early period of the research, so that one might feel that there is nothing left in the nuclear rotation for further study. Against such a negative perspective, I would like to attempt a refutation in this paper that the nuclear rotation still provides something new and interesting.

\section{Classical and Quantum Descriptions of Rigid-Body Motions}

A classical rigid body has six degrees of freedom in total, of which three are responsible for the centre-of-mass motion while the remaining three are for rotation. Thanks to the Galilean invariance, the former three can be neglected from the study of rigid-body dynamics, as far as no external forces are imposed onto the rigid body. The remaining three degrees of freedom are thus regarded as the essential dynamical variables. Due to the nature of classical rigid body, the orientation of the object can be uniquely specified with the three orientational angles $\Omega=\left(\Omega_{1}, \Omega_{2}, \Omega_{3}\right)$, such as the Euler angles, which corresponds to the dynamical variables for the rigid-body rotation. Thus, the time evolution of $\Omega$ tells us how the rigid body makes rotations in time. In classical mechanics, the time evolution is obtained through the Euler equation, a second-order differential equation with respect to time.

Quantization of the rigid-body rotation was formulated by Casimir and Wigner [2]. The rotational motion is expressed in terms of the so-called " $D$ functions" as the quantum-mechanical wave function. The corresponding eigenvalue equation reads $\hat{H}_{\text {rot }} \Psi_{I M K}(\boldsymbol{\Omega})=E_{M K}^{I} \Psi_{I M K}(\boldsymbol{\Omega})$, where the Hamiltonian is given

$$
\hat{H}_{\text {rot }}=\frac{\hat{I}_{1}^{2}+\hat{I}_{2}^{2}}{2 \mathcal{J}_{0}}+\frac{\hat{I}_{3}^{2}}{2 \mathcal{J}_{3}}
$$

for a rigid body with the axial symmetry, and the wave function is expressed as

$$
\Psi_{I M K}(\boldsymbol{\Omega})=\sqrt{\frac{2 I+1}{8 \pi^{2}}} D_{M K}^{I *}(\boldsymbol{\Omega}) .
$$

Wigner considered the Euler angles as the dynamical variables $\boldsymbol{\Omega}=(\alpha, \beta, \gamma)$ to define the $\mathrm{D}$ functions $D_{M K}^{I}(\alpha, \beta, \gamma)$. Details of the $\mathrm{D}$ functions are available, for example, in Ref. [3]. There are three quantum numbers $I, M, K$, in correspondence to three dynamical variables $\boldsymbol{\Omega}$.

Unlike its classical counterpart, the quantum-mechanical rigid body shows "blurred" orientation, to be consistent with Heisenberg's uncertainty principle, so that its motion is described not in terms of the explicit time dependence of 
the dynamical variable $\Omega$, but the $\mathrm{D}$ function, that is, the probability amplitude to find the orientation of the rigid body to be $\Omega$.

Quantum mechanical ways to describe dynamical motions can be also seen in the description of the simple harmonic oscillator, for instance. The classical solution of the simple harmonic oscillation is the trigonometric function, and its velocity becomes maximum in the bottom of the parabolic potential. On the contrary, the ground state of the simple harmonic oscillator in quantum mechanics is the Gaussian, which implies the small oscillation in the vicinity of the bottom of the potential. From a classical point of view, the ground state should be regarded as the oscillator at rest in the bottom of the potential. The width of the Gaussian describes the uncertainty principle, which means that the exact position cannot be specified.

In a similar way, the orientation of the rigid body cannot be specified accurately once quantization is carried out. The orientation should be "fluctuated" due to the uncertainty principle. However, due to the isotropy of the three-dimensional space, there are the countless numbers of the ways to place the non-rotating rigid body in space. These non-rotating states are all energetically degenerate. In other words, the fluctuation amplitude is expected to be so large. This situation is well described by $D_{00}^{0}(\Omega)=1 / \sqrt{4 \pi}$, which can be interpreted as a superposition of rigid bodies at rest with different orientations with the equal probability. This superposition is related to the Nambu-Goldstone boson induced by the spontaneous symmetry breaking in the quantum field theory.

\section{Linear Rigid Body}

The total degrees of freedom of a rigid body is six, as mentioned above. There are numerous proofs for this remark, but the simplest way is to consider many particles connecting to the fixed distance.

One point particle has three degrees of freedom in three-dimensional space. Two particles connected to the fixed distance have five degrees of freedom because two particles carry six degrees of freedom $(6=3 \times 2)$ but the distance condition reduces the degrees of freedom by one $(5=6-1)$. Three particles connected to the fixed distances have similarly calculated to have six degrees of freedom $(6=3 \times 3-2)$. When a rigid body is considered as a collection of many particles connected to the fixed distances, one can demonstrate that the total degrees of freedom is always six for the systems consisting of more than two particles. This result is generalised to conclude that a rigid body has six degrees of freedom.

Molecular physics or physical chemistry sometimes consider a "linear rigid body" of five degrees of freedom, because they need to consider rotational motions of diatomic molecules such as $\mathrm{O}_{2}$ and $\mathrm{H}_{2}$, which is a special case of the rigid body connecting two particles to the fixed distance.

The linear rigid body spends three degrees of freedom for the description of 
the centre-of-mass motion, hence there are only two degrees of freedom left for orientation. Intuitively, the linear rigid body is a straight string or line with a finite length, so that rotational angle around the symmetry axis is redundant. This means that the active dynamical angles can be the polar and azimuthal angles $(\theta, \phi)$.

In quantization, only one commutable pair is necessary, so that $\hat{I}^{2}$ and $\hat{I}_{Z}$ can be chosen. In this case, one can demonstrate that the $\mathrm{D}$ function reduces to the spherical harmonics,

$$
D_{M 0}^{I}(\phi, \theta, 0)=\sqrt{\frac{4 \pi}{2 I+1}} Y_{I M}^{*}(\theta, \phi),
$$

where $\theta$ and $\phi$ correspond to $\beta$ and $\alpha$ in the Euler angles.

This situation provides an interesting situation because the spherical harmonics is usually regarded as the "shape" of the quantum many-body systems. For instance, the pairing-plus-quadrupole model, a self-consistent mean-field model extended from the Nilsson model, assumes the quadrupole shape for the mean field, which is expressed with help of the spherical harmonics $Y_{2 M}(\theta, \phi)$.

In the case of the linear rigid body, the "shape" and "orientation" are expressed through the common mathematical functions, by chance. But this situation can produce a physically interesting problem of nuclear rotation.

\section{Nuclear Cases of the Linear Rigid-Body Approximation}

One may easily imagine that a deuteron can be a nuclear example of the linear rigid body, the system of a proton and a neutron. However, the nucleus does not possess excited states. Only the bound ground state is known.

The second candidate may be the nuclear system of two alpha particles, which is Beryllium $8\left({ }^{8} \mathrm{Be}\right)$. Beryllium 8 has rotational bands and other excited states, but the ground state of ${ }^{8} \mathrm{Be}$ is not bound against a separation to two alpha particles by just $0.092 \mathrm{MeV}$.

The nearly-bound ground state of ${ }^{8} \mathrm{Be}$ caught interests of many physicists in various branches of physics. For example, astrophysics pays attention to this nucleus. ${ }^{8} \mathrm{Be}$ is the gateway to the He burning process in the nucleosynthesis in stars. The primary state of the nucleosynthesis is the pp chain, a nuclear fusion among hydrogen nuclei (or protons) in stars. When hydrogen gas runs out, the gravitational collapse happens to increase the thermal energy of alpha particles, which are a product of the pp chain. The fusion of alpha particles is the entry point to the secondary stage of the nucleosynthesis, that is, the He burning process to produce ${ }^{12} \mathrm{C},{ }^{16} \mathrm{O}$, etc. The fact that the ground state of two alpha particles is unbound means that the fusion of alpha particles must be more rapid than the rate of the separation to two alpha particles. This condition narrows the possibility of the onset of the He burning in many stars. At present, the presence 
of the Hoyle states in ${ }^{12} \mathrm{C}$, a resonance of three alpha particles, are suggested to help the entry to the He burning process. However, if some physical constants such as the pion mass or its coupling constants to baryons, are slightly different from the values in the present universe, a totally different "look and face" can be seen in one of the universes in the multiverse theory [4] because the parameter shifts might stabilise the bound between two alpha particles.

It is worth an examination whether the shape of ${ }^{8} \mathrm{Be}$ allows the linear rigidbody approximation. From the alpha-cluster calculations [5], the shape of ${ }^{8} \mathrm{Be}$ is reported to be a dumbbell-like shape: the size of elongation is about $3 \mathrm{fm}$, while the diameter of the alpha particle was experimentally observed in 2021 to be $1.67824 \times 2=3.35648 \mathrm{fm}$ [6]. A substantial overlap is expected between the alpha particles, so that the linear rigid-body approximation seems to be a bit drastic for ${ }^{8} \mathrm{Be}$. Keeping this in mind, let us explore an application of the linear rigid-body approximation to ${ }^{8} \mathrm{Be}$.

\section{Rotational-Band Members}

Now, I would like to apply the Bohr-Mottelson Hamiltonian, Eq. (1), to the linear rigid-body approximation of ${ }^{8} \mathrm{Be}$ as a two-alpha compound system. The eigenstates of Eq. (1) is expressed as a direct product of the collective and intrinsic states, because these degrees of freedom are decoupled in the Hamiltonian.

Because the D function reduces to the spherical harmonics, the linear rigid-rotor approximation does not distinguish the collective and intrinsic parts except the vibrational part $R_{n l}(r)$ permitting the oscillation of the alpha particles. The collective dynamical variables and the intrinsic dynamical variables are common for the orientation $(\theta, \phi)$ because the relative angular position of the second alpha particle to the first is identical to the orientation of the linear rigid body.

The collective part gives rise to

$$
\hat{H}_{\mathrm{coll}} Y_{M}^{I}(\theta, \phi)=I(I+1) \hbar^{2} Y_{M}^{I}(\theta, \phi) .
$$

The intrinsic part gives

$$
\hat{H}_{\mathrm{intr}} R_{n l}(r) Y_{m}^{l}(\theta \phi)=l(l+1) \hbar^{2} R_{n l}(r) Y_{m}^{l}(\theta, \phi) .
$$

The band head $0^{+}$state is exclusively expressed by the intrinsic state,

$$
\left(0^{+}\right) \propto R_{10}(r) Y_{0}^{0}(\theta \phi) \propto R_{10}(r)
$$

where the last step is obtained because $Y_{0}^{0}$ is a constant. If the Beryllium 8 is stiff and well approximated as the linear rigid body, the radial wave function must be a narrow Gaussian-like function, which corresponds to the small oscillation of the alpha particles with respect to the rigid-body limit ( $r \simeq 3 \mathrm{fm}$ according to the numerical calculation [5]). 
The rotational states of ${ }^{8} \mathrm{Be}$ were so far observed up to $4^{+}$although the $2^{+}$was discovered relatively recently [7]. These band members are expressed as

$$
\left(2^{+}\right) \propto R_{10}(r) Y_{2 M}(\theta, \phi), \quad\left(4^{+}\right) \propto R_{10}(r) Y_{4 M}(\theta, \phi) .
$$

Note that the radial part is fixed to the band-head state because the adiabatic condition is assumed for rotational-band members.

\section{Numerical Estimate for the Radial Function}

The radial function obtained in the intrinsic Hamiltonian carries information how good the linear rigid rotor approximation is. When this approximation can be justified, a narrow Gaussian-like function should be obtained numerically.

As usually done, the intrinsic wave function $\Psi_{\text {intr }}$ is decomposed to

$$
\Psi_{\text {intr }}=R_{n l}(r) Y_{l m}(\theta, \phi),
$$

where the relative coordinate between the two alpha particles is expressed in the three-dimensional polar coordinates $(r, \theta, \phi)$. When the radial function is expressed as $R_{n l}(r)=u_{n l}(r) / r$, the intrinsic part of the Hamiltonian $\hat{H}_{\text {intr }}$ is expressed as a one-body Schrödinger equation for $u_{n l}(r)$,

$$
\hat{H}_{\mathrm{intr}}=-\frac{\hbar^{2}}{m_{\alpha}} \frac{d^{2}}{d r^{2}}+\frac{l(l+1) \hbar^{2}}{m_{\alpha} r^{2}}+\hat{V}(r)
$$

The reduced mass $\mu=m_{\alpha} / 2$ is already inserted in the above equation. The twobody interaction $\hat{V}$ is considered as the phenomenological interaction between two alpha particles, which has been first developed by Buck, et al. [8]. The Buck potential consists of the Gaussian for the nuclear interaction and the error function for the Coulomb interaction.

In the present work, we approximate the Buck potential with multiple squarewell potential for the sake of simplicity. In the absence of the centrifugal barrier, the potential is set to

$$
\begin{aligned}
V_{\mathrm{sq}}(\rho) & =-\mathcal{V}_{0}=-15 \quad(0<\rho<2.5) \cdots & & \text { Region I: } \\
& =\mathcal{V}_{1}=1 \quad(2.5<\rho<8) \cdots & & \text { Region II: } \\
& =0 \quad(8<\rho) \cdots & & \text { Region III: },
\end{aligned}
$$

where $\rho$ is the scaled radius $\left(\rho=r / r_{0}\right.$, where $r_{0}=1.5 \mathrm{fm}$ ) and the unit of energy is scaled with $E_{0}=\hbar^{2} /\left(m_{\alpha} r_{0}^{2}\right)=4.628 \mathrm{MeV}$.

The scaled eigenvalue equations are solved with the following wave functions

$$
\begin{aligned}
u(\rho) & =A \sin (k \rho) \cdots & & \text { Region I, } \\
& =B_{1} \exp (q \rho)+B_{2} \exp (-q \rho) \cdots & & \text { Region II, } \\
& =C_{1} \exp \left(i k_{0} \rho\right)+C_{2} \exp \left(-i k_{0} \rho\right) \cdots & & \text { Region III. }
\end{aligned}
$$


The eigenvalue equation, which can be obtained through the continuity conditions among the above radial equations, reduces to

$$
\frac{\tanh (q(d-1))}{q}=\frac{k \sin \left(2 k_{0} d\right)-k-k_{0} \tan k \cos \left(2 k_{0} d\right)}{q^{2} \tan k\left\{1-\sin \left(2 k_{0} d\right)\right\}+k k_{0} \cos \left(2 k_{0} d\right)},
$$

where $d=b / a . k, q$ and $k_{0}$ are the parameters to be numerically determined.

It should be noted that the energy eigenvalue must be positive $\left(\mathcal{E}_{0}>0\right)$ because all the states of ${ }^{8} \mathrm{Be}$ are regarded as resonances. Unlike the bound-state eigenvalue problems, we cannot make use of the square-integrable conditions to calculate the resonant wave functions. The wave functions in the Region III extends up to the infinity, as seen in Eq. (13). This aspect is numerically so challenging, that applications of the "bound-state" approximation is seen in many literatures so as to obtain resonance states. In the present paper, however, a "genuine" resonant states are numerically obtained by virtue of the square potential.

In Figure 1, calculated wave functions of resonance are depicted. The upper figures are for the "ground" state (the small window on left is the magnification of the Regions I and II) and the lower figure is for the "excited" state.

As considered above, this quantum-mechanical framework without time depen-

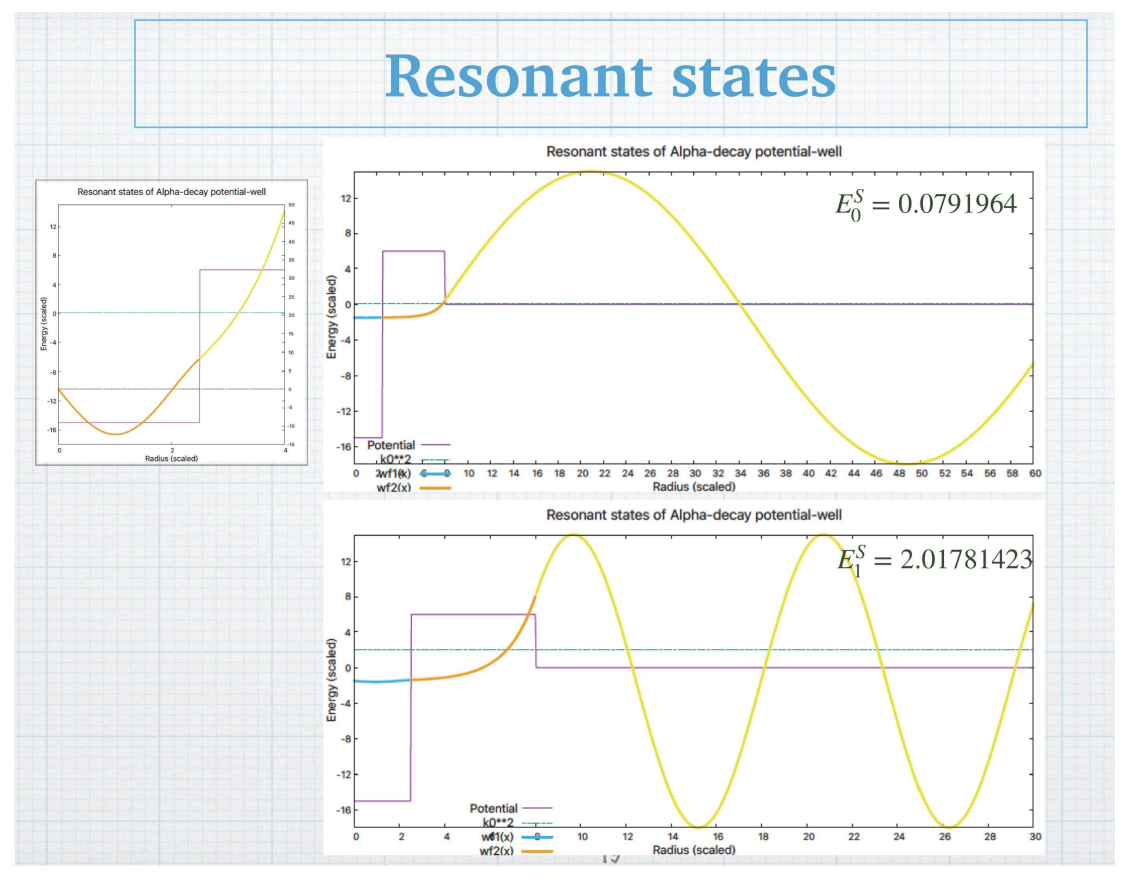

Figure 1. (Color online) Numerically obtained resonant states for the square potential, approximated to the Buck potential. 
dence superposes all the motions in the classical picture. Therefore, the continuum states, which correspond to the particle escaping from the potential, have more probability amplitudes than the confined (resonant) states inside the potential. Nevertheless, the magnified view inside the potential shows that the wave function has a form similar to the Gaussian, although the width is not so narrow as expected. Therefore, the linear rigid approximation cannot be perfectly justified from this numerical calculation, but may be acceptable with a certain compromise.

There are some claims that this type of "genuine" resonant states are blurred in its physical meaning due to too much components in the continuum part (Region III). Our physical understanding of the resonance is that the wave packet is more confined inside the potential for a certain limited time, and then decays into the continuum. To emphasize such a picture, there are some works to multiply a damping factor to the wave function, $\exp \left(-\eta \rho^{2}\right) u(\rho)$. With the value $\eta=0.2$ from Ref. [9], the radial functions are redrawn in Figure 2.

With this manipulation, the physical image about the resonance is improved and the linear rigid-body approximation looks better. It is certain that there are plenty of room to make further progresses in handling the resonant states numerically and theoretically.

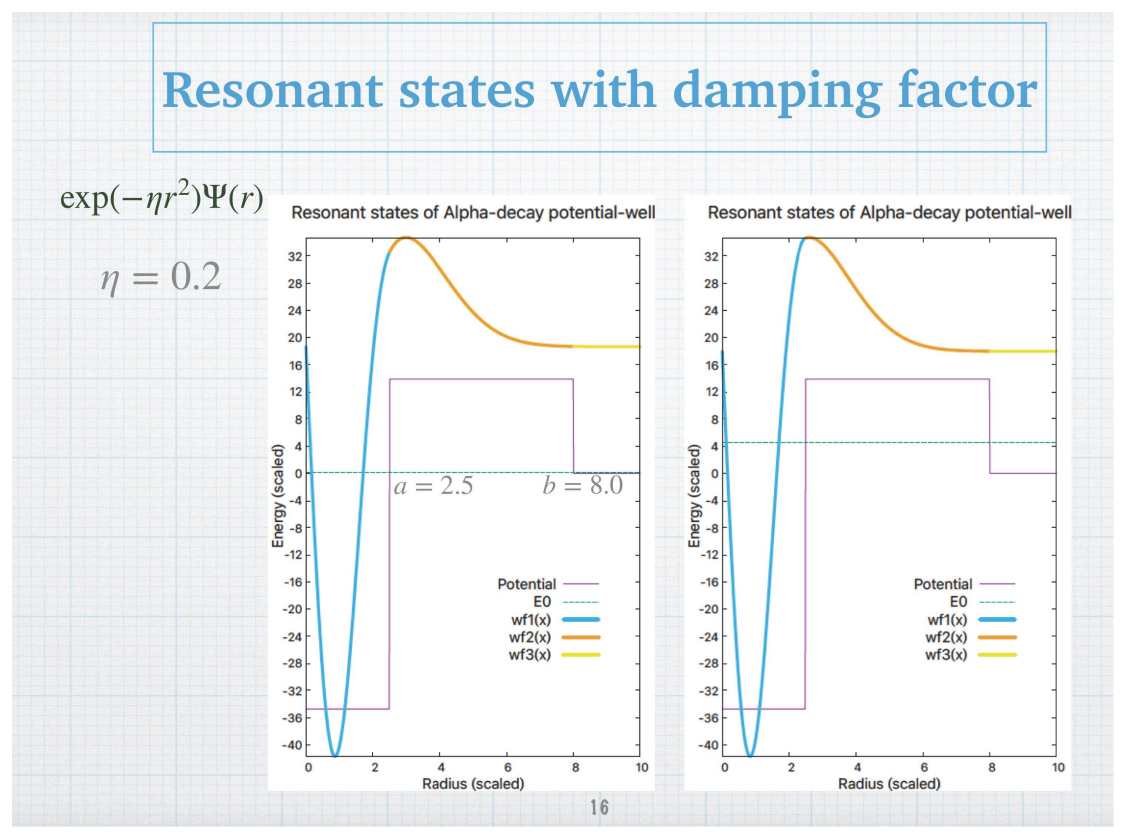

Figure 2. (Color online) The radial functions with the damping factor. The left and right panels are for the ground and excited states, respectively. 


\section{Rotation-Resonant Coupling Model}

In the linear rigid-body approximation, the structure of the wave functions are quite similar between the rotational members and the group of resonant states.

As already considered in Eq. (9), the rotational states are a product of the ground state in the intrinsic part and the spherical harmonics originating from the collective rotation. On the contrary, the resonant states are expressed as the intrinsic wave functions, Eq. (10).

From a physical point of view, angular momenta carried by the rotational states and the resonant states can be interpreted in a different way: the angular momenta of the rotational states are obviously due to the collective rotation of the linear rigid body. On the contrary, the angular momenta carried by the resonant states come from the centrifugal barrier to confine the alpha particle. Of course, this difference is of the surface, coming from the speciality of the linear rigidbody. It is clear that the relative motion of the second particle can be interpreted as the rotation and vibration of the "rigid body". From a general theoretical viewpoint, rotation and resonance can be different, but they happen to be the same in the linear-rigid body approximation. Hence we make use of this "similarity by chance" in our theoretical model, which I call "rotataion-resonance coupling model".

Let us consider the $I=2$ states in ${ }^{8} \mathrm{Be}$, as an example of the rotation-resonance coupling. The rotational and resonant states are expressed as $R_{10}(r) Y_{2 M}(\theta, \phi)$ and $R_{12} Y_{2 M}(\theta, \phi)$, respectively. These states are eigenstates of the BohrMottelson Hamiltonian, Eq. (1), and their energies are respectively given as

$$
E_{\text {rot }}^{2+}=\mathcal{E}_{10}+\frac{2 \cdot(2+1) \hbar^{2}}{2 \mathcal{J}_{0}}, \quad \text { and } \quad E_{\text {res }}^{2+}=\mathcal{E}_{12}+\frac{2 \cdot(2+1) \hbar^{2}}{2 \mathcal{J}_{0}}
$$

These states contains the same angular dependency, that is, the spherical harmonics, but they are orthogonal to each other in the radial sector. As mentioned earlier, the Bohr-Mottelson Hamiltonian presents the ideal situation where the rotation and vibration are decoupled (or adiabatic rotation with respect to the intrinsic state). In reality, however, these collective modes might be coupled (or diabatic). Let us assume such a coupling in the Bohr-Mottelson Hamiltonian as $\hat{H}_{\text {rot-res }}=\hat{H}_{B M}+\hat{V}_{\text {rot-res }}$. The coupled state is regarded with the coupling constants as

$$
\Psi_{I M}=\left(c_{\text {rot }} R_{10}(r)+c_{\text {res }} R_{12}(r)\right) Y_{2 M}(\theta, \phi) .
$$

The eigenvalue equation reduces to the well-known two-state problem, of which the relevant equation reads

$$
\left(\begin{array}{cc}
V_{11}+E_{\text {rot }}^{2+} & V_{12} \\
V_{21} & V_{22}+E_{\text {res }}^{2+}
\end{array}\right)\left(\begin{array}{c}
c_{\text {rot }} \\
c_{\text {res }}
\end{array}\right)=E_{\text {rot-res }}^{2+}\left(\begin{array}{c}
c_{\text {rot }} \\
c_{\text {res }}
\end{array}\right),
$$


where the perturbed energy is expressed as

$$
E_{\text {rot-res }}^{2+}=\mathcal{E}_{10}+\frac{6 \hbar^{2}}{2 \mathcal{J}_{0}}+\frac{1}{2}\left(\mathcal{E}_{12}-\mathcal{E}_{10}+V_{11}+V_{22} \pm \Delta E\right) .
$$

The energy splitting is given as

$$
\Delta E=\sqrt{\left(V_{22}-V_{11}\right)^{2}+2\left(V_{22}-V_{11}\right)\left(\mathcal{E}_{12}-\mathcal{E}_{10}\right)-4 \mathcal{E}_{10} \mathcal{E}_{12}+4 V_{12}^{2}} .
$$

The coupling term considered here is so far purely theoretical and merely an assumption. We need to look at experimental data whether such coupling is present in reality or not. Figure 3 is the energy spectrum of ${ }^{8} \mathrm{Be}$. There is an M1 transition from the second $2^{+}$state (at $16.626 \mathrm{MeV}$ ) to the first $2^{+}$state (at $3.030 \mathrm{MeV}$ ), implying a possibility of the presence of the rotation-resonance coupling. According to the rotation-resonance model, the first $2^{+}$state has a more rotational character than the second, which is expected to be of a resonant character. Based on this experimental data, the coupling constant may be numerically determined and the result can be used for a prediction of a possible transition from the second $4^{+}$state to the first $4^{+}$. This line of study is under construction.

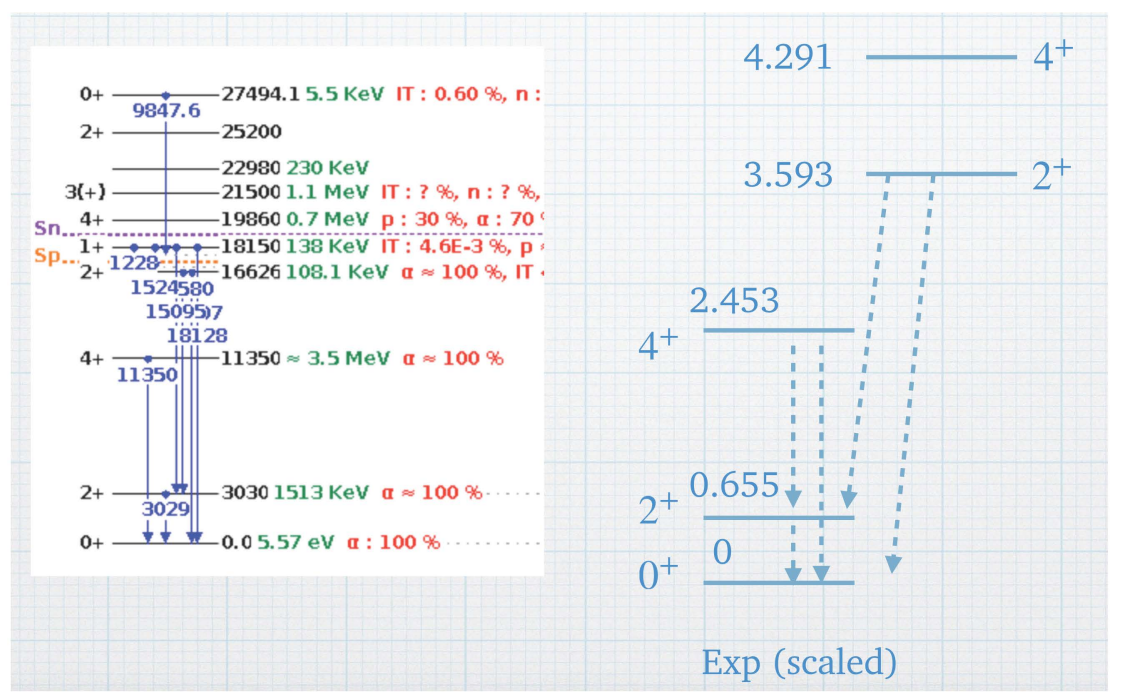

Figure 3. (Color online) Rotational spectra and other excited states of ${ }^{8} \mathrm{Be}$. Data source: Brookhaven National Laboratory (nndc.bnl.gov)

\section{Conclusion}

Nuclear rotation is reviewed and reanalysed with the linear rigid-body approximation. The approximation was applied to the Beryllium 8 nucleus, where the 


\section{Revisiting the Concept of Nuclear Rotation}

ground state is unbound. The resonant states are numerically examined and a possible coupling between the rotational and resonant states built upon the unbound state is discussed. A further study is need to quantify the coupling constant and to improve the numerical expression of the resonant states. In addition, the two-body potential needs to be upgraded from the multi-square-well potential to the Buck potential, which is more realistic.

\section{References}

[1] A. Bohr, B. Mottelson (1975) "Nuclear Structure", Vol. II. Benjamin.

[2] H.B.G. Casimir (1931) Rotation of a rigid body in Quantum Mechanics. PhD thesis; E. Wigner (1931) "Gruppentheorie und ihre Anwendungen auf die Quantenmechanik der Atomspektren". Friedrich Vieweg und Sohn, Braunschweig.

[3] M.E. Rose (1957) “Elementary Theory of Angular Momentum”. John Wiley \& Sons, New York.

[4] F.C. Adams, E. Grohs (2017) Astropart. Phys. 8740.

[5] T. Yoshida, et al. (2013) J. Phys.: Conf. Ser. 454012053.

[6] J.J. Krauth, et al. (2021) Nature 589527.

[7] V.M. Datar, et al. (2005) Phys. Rev. Lett. 94122502.

[8] B. Buck, H. Friedrich, C. Wheatley (1977) Nucl. Phys. A 275246.

[9] E. Garrido, et al. (2013) Phys. Rev. C 88024001. 